\title{
The effect of radiotherapy on rectal cancer: a histopathological appraisal and prognostic indicators
}

\author{
Mohammad AlQudah', Emil Salmo², Najib Haboubi ${ }^{3}$ \\ ${ }^{1}$ Department of Pathology and Microbiology, School of Medicine, Jordan University of Science and Technology, Irbid, Jordan \\ ${ }^{2}$ Department of Histopathology, The Pennine Acute Hospitals NHS Trust, The Royal Oldham Hospital, Oldham, UK \\ ${ }^{3}$ Department of Histopathology, Spire Manchester Hospital, Manchester, UK
}

Received: January 14, 2020

Revised: April 7, 2020

Accepted: April 13, 2020

\section{Correspondence:}

Mohammad AlQudah, Department of Pathology and Microbiology, School of Medicine, Jordan University of Science and Technology, Irbid, Jordan.

Tel: +962-799999571

Fax: $+962-2-7200638$

E-mail: m.alqudah12@just.edu.jo

ORCID:

https://orcid.org/0000-0001-6877-1184
The management of rectal cancer is a major undertaking. There are currently multiple treatment modalities with variable degrees of complications. Radiotherapy (RT) is one of the more frequently used modalities either on its own or more frequently with chemotherapy mostly before the definitive surgery. The outcome of RT is unpredictable. RT has its serious side effects and there are no guarantees of its usefulness in all patients. This article outlines the effect of RT on the tumor, reviews the various staging systems of responses to RT and present recent evidence of which case is less responsive to such treatments to avoid unnecessary complications.

Keywords: Rectal cancer, Therapeutic oncology, Clinical pathology, MicroRNAs, Regression, Chemoradiotherapy

\section{Introduction}

Rectal cancer is one of the more frequent human malignancies accounting for $3.9 \%$ of all new cancer cases causing $3.2 \%$ of all cancer-related deaths globally in 2018 [1]. The survival of the disease increased in the last years reaching an overall 5-year rate of 67\% [2]. This could be related to the medical advances in rectal cancer treatment, increased awareness and screening [3].

Surgery is still the mainstay of treatment for rectal cancer since the introduction of abdomino-perineal resection techniques in 1908 [4]. Since then advances progressed the surgical platform and total mesorectal excision (TME) became the standard surgical approach ever since its introduction by Heald and Ryall [5] in 1982.

Neoadjuvant and adjuvant therapies have been repeatedly tested in many trials to improve the outcomes of rectal cancer patients in terms of local recurrence and survival. The addition of radiotherapy (RT), in addition to the improvement in surgical techniques, demonstrated improvement in local recurrence $[6,7]$. Despite that, other studies reported that around one-fifth of the cases will have no response to RT. Other workers reported that the contribution of neoadjuvant RT to pathological complete response in terms of survival was of limited or minimal benefit [8].

In view of the above changes, the real challenge in rectal cancer treatment is to identify patients who would fully benefit from the new treatment protocols which involve RT. The aim of this article is to present the different histological systems used to classify the response rate of the patients and to discuss the different types of biomarkers used to predict of the response of patients to RT/ chemoradiotherapy (CRT).

\section{RT Mechanism of Action}

Oxidative stress damage is extensive in cells exposed to ionising radiation. This damage mainly targets nuclear DNA through inhib-

Copyright@ 2020 The Korean Society for Radiation Oncology

This is an Open Access article distributed under the terms of the Creative Commons Attribution Non-Commercial License (http://creativecommons.org/licenses/by-nc/4.0/) which permits unrestricted non-commercial use, distribution, and reproduction in any medium, provided the original work is properly cited. 
iting DNA transcription in both direct and indirect pathways [9]. DNA damage is caused by the direct targeting nuclear chromatin. This damage will find the way through generating intra- and inter-strand chromosomal cross linkages along with breaks and mutations of DNA material. Direct damage to plasma membrane happens by disrupting both electrical gradient and the rigidity of the two layers of phospholipids membranes affecting the cell integrity. The indirect pathway is through free radicals from the ionisation of $\mathrm{H}_{2} \mathrm{O}_{2}$ molecules [10].

Altering the coordinated repair of DNA processes that repair damage induced by ionising radiation improves outcome. Cell repair, at low radiation level, can accommodate injuries such as double strand breaks and with increasing level the inflicted damage on the cell promote the cells to enter apoptosis and eventual cell death. This sensitivity to the dose of radiation that inflicts these injuries differs from tumor to tumor and the surrounding non-tumorous tissues [11]. Cells with high turnover are typically the first to respond to radiotherapy treatment thus fulfilling the aim of RT which is to eradicate or at least debulk the tumor so it becomes more amenable to surgical excision [12].

The addition of chemotherapy to the treatment plan has additional effects on cell's response to radiation and this combined treatment leads to either delay or abolition of the reparative process. These chemotherapeutic agents will target the cell cycle at more sensitive phase [13]. The flurouracil-based neoadjuvant CRT (nCRT) was further improved by adding oxaliplatin. This regimen significantly improved disease-free survival of patients with stages cT3-4 or cN1-2 rectal cancer in comparison to the older regimen [14].

In 2004, Habr-Gama et al. [15] have pioneered the concept of "wait and see" for rectal cancer as they have found significant number of patients who have received CRT responded very well and staged as complete pathological responders. As those patients have an excellent prognosis according to Habr-Gama et al. [15], they proposed that carefully selected rectal cancer patients responding to CRT should have a nonsurgical management. Despite the argument against wait and see [16] which stated that the rationale of a "wait and see" policy when complete clinical response status is achieved relies on retrospective observations, and data are currently insufficient to support this policy except in patients who are recognized to be unfit for or refuse radical surgery. The "wait and see" policy however is gaining ground and many centres in the world are following the Sao Paulo regime with great success as it has shown excellent outcomes in 25\%-40\% of carefully selected and staged patients [17].

The challenge we are facing now is how to accurately predict pathological complete response ( $\mathrm{pCR}$ ) to change the management plan before radical surgery. An increased interest in assessment of $n C R T$ response is well documented, but this did not show much success yet. In the "watch-and-wait" approach, patients deemed to have achieved clinical complete response (cCR) must meet strict inclusion criteria. This includes digital rectal examination, direct visualization by colonoscopy, and biopsying suspicious lesions in the irradiated tumor bed [18]. Difficulty in distinguishing fibrosis from residual tumor and the distribution of residual rectal cancer within different layers of bowel wall after $\mathrm{nCRT}$ poses a significant difficulty in assessing complete response [19]. The use of positron emission tomography (PET) as an addition for the clinical assessment of $\mathrm{cCR}$ is a refinement that improved the overall accuracy from $91 \%$ to $96 \%$ [20].

\section{Histological Effect of RT on the Neoplastic Cells}

In 1989, a prospective study of 186 rectal carcinoma patients was carried out, in which, 97 were randomized to surgery alone and 89 to surgery with preoperative radiotherapy [21]. The study showed that radiation under-staged the disease biologically but over graded it histologically in the sense that the appearances of individual cells may appear more bizarre after CRT than before treatment. It also suggested that any clinical pathology staging should include the presence or absence of radiation. To that extent the minimum dataset (MDS) which is used for all cancers in UK stipulated that the form should state whether the tumor received radiation therapy and if so whether there is response. The response is determined by looking into the volume of the neoplasm versus fibrosis. The MDS categorise the response rate as follows: no residual tumor/ mucous lakes only, minimal residual tumor or no marked regression [22].

\section{Staging Systems}

Over the years, many systems for tumor regression grading with various scoring parameters were developed. The first was developed in 1994 by Mandard et al. [23], it was designed to assess tumor response in oesophageal cancer patients treated with nCRT. They used a five-tier system to classify regression from score 1 (no tumor cells; complete regression) to score 5 (no regression). The main advantage of the Mandard's Tumor Regression Grade (TRG) system is comparing the proportion of the residual cancer cells to the amount of fibrosis. This was considered simple and was shown to be a reproducible method; 3 years later it was modified to colorectal cancer specimens by Dworak et al. [24] in 1997. They classified regression into five grades but the other way around from 0 (no re- 
gression) to 4 (total regression). In 2002, Wheeler et al. [25] devised a three-point grading system called rectal cancer regression grade, where grade 1 indicated complete response, grade 2 showed marked fibrosis despite persistent microscopic disease, and grade 3 showed no response with little or no fibrosis taking into consideration the macroscopic features of the specimen. This was later modified by Bateman et al. [26], in which both extremes were preserved; complete replacement of viable cancer cells by fibrosis and cancer cells persistence without fibrotic changes but subtle changes were added for groups of near-complete responders. Cutoffs of 0\%-5\%, 5\%-50\%, and >50\% residual tumor were adopted. Although several grading systems for tumor response have been devised, a three-point TRG has been shown to provide good inter-observer reproducibility compared to the five grades, but both systems were shown to provide similar prognostic significance. The three-point system devised in Ryan et al. [27] has been shown to be reproducible and easy to use, with good inter-observer agreement. The most recently published classification by the Royal College of Pathologists dataset guidelines for colorectal cancer (2017) and the College of American Pathologists based on the AJCC/UICC, 8th edition [28] recommend a four-tier system with modification to the one described by Ryan et al. [27]. This is considered the best methodology and provided excellent inter-observer reproducibility compared with five-grade systems. It also showed similar prognostic significance. Table 1 summarizes staging systems by Wheeler et al. [25], Mandard et al. [23], and AJCC/UICC 8th edition. More recently however a group from South Korea compared Ryan et al. [27]'s, AJCC and modified Dworak staging systems and found that the modified Dworak (which included the assessment of mural tumor and the lymph node status) as a best system to reflect recur- rence and disease free survival [29].

Mucin pools are a common feature during histopathological assessment of specimens treated with $\mathrm{nCRT}$ but the presence of acellular mucin following nCRT does not have a significant impact on patient outcome. Only the presence of malignant cells in the specimen is the considered criteria for staging of tumor following preoperative treatment. Haemorrhage, necrosis, inflammation and acellular mucin are not considered as part of staging process, they are considered only for studying regression [22].

In a study by Fokas et al. [30] in 2018, the prognostic role and surrogacy of the neoadjuvant rectal (NAR) score was examined for $c T, y p T$ and ypN categories. NAR scores were categorised into three degrees; low when the score is below 8, intermediate (score between 8 and 16) and high in scores over 16 based on the observed scores in a clinical trial. In the previous study, NAR scores were capable of predicting treatment effects on clinical outcome and overall survival with lower NAR scores having better cumulative incidence of disease free survival, overall survival, and distant metastasis [30].

\section{Types of Radiotherapy}

The commonest modalities for RT are short course (SC) followed by surgery after 5 days, SC with extended interval, long course (LC) combined with chemotherapy and the contact radiotherapy. Contact radiotherapy, also known as Papillon treatment, is a modality of radiotherapy given from inside the rectum in low doses reaching only few millimetres into the tissue. SC is given as 25 Gy over 5 days with a daily dose of $5 \mathrm{~Gy}$. LC is given as a total dose of 45-50 Gy in 25-28 fractions [31].

Table 1. Grading systems

\begin{tabular}{|c|c|c|}
\hline & Grade & Description \\
\hline \multirow[t]{5}{*}{ Mandard's Tumor Regression Grade [23] } & 1 & No residual cancer \\
\hline & 2 & Rare residual cancer cells \\
\hline & 3 & Fibrosis outgrowing residual cells \\
\hline & 4 & Residual cancer outgrowing fibrosis \\
\hline & 5 & Absence of regressive changes \\
\hline \multirow[t]{3}{*}{ Rectal Cancer Regression Grade (RCRG) [25] } & 1 & $\begin{array}{l}\text { Pathological complete response or only microscopic foci of adenocarcinoma re- } \\
\text { maining }\end{array}$ \\
\hline & 2 & Marked fibrosis but macroscopic disease is present \\
\hline & 3 & Poor response with little or no fibrosis and abundant macroscopic disease \\
\hline \multirow[t]{4}{*}{ AJCC, Royal College of Pathologists four-tier system } & 0 & Complete response: No viable tumor cells \\
\hline & 1 & Near-complete response: Single cell or rare small groups of cancer cells \\
\hline & 2 & $\begin{array}{l}\text { Partial response: Residual cancer with evident tumor regression but more than sin- } \\
\text { gle cells or rare small groups of cancer cells }\end{array}$ \\
\hline & 3 & Poor or no response: Extensive residual cancer with no evident tumor regression \\
\hline
\end{tabular}

AJCC, American Joint Committee on Cancer. 
Comparing SC to LC radiotherapy, both had similar outcomes in terms of sphincter preservation, local recurrences, late toxicity, the overall survival, disease-free survival and quality of life. However, pCR was higher in patients receiving LC of RT $[32,33]$. There is no uniformity of using SC or LC as practice varies widely but in many centres the use of $\mathrm{LC}$ is reserved when the circumferential resection margin (CRM) is threatened, otherwise SC is the preferred treatment. In other centres they only use SC or LC alone. Now preoperative RT or CRT is mostly used as it is less toxic and more efficient than postoperative RT [34]. There is very little experience on the histological changes after contact therapy or SC with extended interval.

\section{Preoperative vs. postoperative CRT}

The timing of radiotherapy with the administration of chemotherapy treatment is crucial to accomplish the maximum effect of this therapy [35]. Preoperative CRT was previously reported as the preferred method of treatment for patients with locally advanced rectal cancer. It is associated with improved compliance to CRT and down-staging which plays a role in enhancing the rate of curative surgery as well as doubling the chances of sphincter preservation compared to postoperative radiation in low-lying tumors although in one paper looking at series of stage 3 rectal cancer it was found that there was improvement of local recurrence rate but no overall survival benefit was associated with preoperative compared to postoperative RT [36]. Preoperative radiation was further associated with more effective results due to better tumor oxygenation preoperatively [37].

Preoperative RT might effectively treat systemic micrometastases reducing the rate of local failure. Some modalities of preoperative CRT further showed disappearance of mutations which could be related to the effect of CRT as well as the emergence of new resection mutations which were thought that may be due to either treatment-driven selection or expansion of pre-existing clones which were undetectable due to their low levels. Thus, evidence of intramural heterogeneity in rectal cancer was reported [38].

The other important area is whether SC RT is associated with decrease locoregional recurrence is still associated with stage improvement. In one paper [39], the group looked at both SC RT and LC CRT which have shown that there is stage improvement even in SC RT. This is in contrast to an earlier work from Nagtegaal et al. [40] when they found that no change in stage occurred with SC RT.

$A$ better prognosis and disease-free survival have been seen in patients with completely excised rectal carcinomas who have complete or marked regression of their tumor when received CRT preoperatively $[39,41]$. In a recent systematic review and meta-analysis of 4,875 patients from 17 studies by Kong et al. [42], a signifi- cant association with overall survival was identified. The $15.9 \%$ of patients showed (pCR), and 29.9\% showed no response.

\section{Predictive Biomarkers for CRT in Rectal Cancer}

Some tumors resist the treatment. Furthermore clinical, histopathological and radiological profiles have been unreliable in predicting which tumors would be responsive to the treatment. With regards to radiological modalities, some studies tried utilizing MRI TRG to assess patients preoperatively in order to modify the treatment strategy. They clustered the cases into good responders in which surgery may be delayed to avoid the associated mortality and morbidity whereas poor responders are advised to undergo additional treatment to help down-staging and early treatment systemic relapse risk [43]. Despite that, there is no consensus on this modality requiring more robust studies. Therefore, it is essential to look for predictive biomarkers that are highly sensitive and specific to avoid unnecessarily giving RT treatment to unresponsive tumors. Molecular biomarkers are used in clinical practice to predict treatment response in various malignancies. Several studies have investigated molecular markers in rectal carcinoma, and their ability to predict a response to CRT.

\section{Molecular Markers in Rectal Carcinoma}

\section{MicroRNAs}

MicroRNAs (miRNAs/miRs) are a class of short non-coding RNA sequences composed of around 22 nucleotides that are involved in the post-transcriptional regulation of gene expression. Various miRNAs have been identified to play a role in carcinogenesis by regulating the transcription of oncogenes and tumor suppressor genes. miRNAs can be studied in tissue preparations and also in peripheral blood which could provide a minimally invasive method to test for predictive biomarkers. Eriksen et al. [44] investigated the expression of miRNAs in formalin-fixed paraffin embedded (FFPE) diagnostic samples of rectal carcinoma using real-time quantitative polymerase chain reaction (RT-PCR). TRG was used to assess the response to treatment. Significant positive correlation was found between the low expression of miR-145 and response to CRT. Another group studied the relationship between miR-194 and response to CRT using RT-PCR and in situ hybridisation on FFPE diagnostic samples [45]. They found high levels of miR-194 to correlate significantly to response to treatment using both methods of testing. Luo et al. [46] demonstrated that the up-regulation of miR-519b-3p is associated with a response to CRT. In addition to tissue-based assays, circulating microRNAs have the potential to 
be used as minimally invasive predictive biomarkers. A Chinese research group identified circulating miR-345 as a possible predictive biomarker for CRT [47]. Overexpression of miR-345 was significantly correlated with poor responsiveness to CRT. Tumors with low expression of miR-345 showed higher sensitivity to radiotherapy.

\section{Protein markers}

Protein biomarkers in tissue preparations have been widely explored, and many were found to be correlated with CRT response and thus can be potentially used as predictive biomarkers. DEK is an oncogene that is overexpressed in many cancers including breast cancer, hepatocellular carcinoma, and melanoma. In a study using microarray of diagnostic rectal cancer specimens in the immunohistochemical analysis of DEK, high expression of DEK was found to be associated with better response to CRT. All the tumors with complete response to the treatment were overexpressing DEK [48]. Another group studied the expression of two immunohistochemical markers: cyclooxygenase-2 (COX-2) which is associated with angiogenesis and proliferation, and apoptosis protease-activating factor 1 (APAF-1). High expression of APAF-1 was associated with a better response to CRT, while high expression of COX-2 was associated with a poorer response to the therapy [49]. Therefore, tumors with increased APAF-1 levels and decreased COX-2 levels showed the best response to CRT. APAF-1 and COX-2 are both potential predictive biomarkers to CRT therapy, independently and combined.

Repetto et al. [50] performed proteomic studies on rectal cancer biopsies using two-dimensional differential in gel electrophoresis (2D-DIGE) to discover predictive biomarkers for rectal cancer treatment. They extracted proteins from RC samples and normal tissues and identified 27 differentially expressed proteins in good versus poor responders. Among these, fibrinogen $\beta$ chain fragment $D$, cathepsin D, actin, serpin B5, serpin B9, and peroxiredoxin-4 were highly expressed in poor responders' group and are potential negative predictive biomarkers to CRT.

\section{Conclusion}

miRNA and protein biomarker are promising as predictive biomarkers for CRT in rectal carcinoma. However, none of these biomarkers have been employed in routine clinical practice. A single biomarker is probably unlikely to have the ability to be predictive with high sensitivity and specificity. A biomarker panel, which could include genetic, epigenetic and protein biomarkers, in combination with clinicopathological and radiological data need to be evaluated together to reach a robust method of predicting therapy outcome, which in turn predicts the prognosis, and informs therapeutic ap- proach.

\section{Conflict of Interest}

No potential conflict of interest relevant to this article was reported.

\section{References}

1. Bray F, Ferlay J, Soerjomataram I, Siegel RL, Torre LA, Jemal A. Global cancer statistics 2018: GLOBOCAN estimates of incidence and mortality worldwide for 36 cancers in 185 countries. CA Cancer J Clin 2018;68:394-424.

2. American Cancer Society. Cancer Facts \&t Figures 2019 [Internet]. New York, NY: American Cancer Society; 2019 [cited 2020 May 1]. Available from: https://www.cancer.org/research/cancer-facts-statistics/all-cancer-facts-figures/cancer-facts-figures-2019.html.

3. Bujanda L, Sarasqueta $C_{1}$ Hijona $E_{1}$ et al. Colorectal cancer prognosis twenty years later. World J Gastroenterol 2010;16:862-7.

4. Miles WE. A method of performing abdomino-perineal excision for carcinoma of the rectum and of the terminal portion of the pelvic colon (1908). CA Cancer J Clin 1971;21:361-4.

5. Heald RJ, Ryall RD. Recurrence and survival after total mesorectal excision for rectal cancer. Lancet 1986;1:1479-82.

6. Pahlman L. Initial report from a Swedish multicentre study examining the role of preoperative irradiation in the treatment of patients with resectable rectal carcinoma. Swedish Rectal Cancer Trial. Br J Surg 1993;80:1333-6.

7. Hyams DM, Mamounas EP, Petrelli N, et al. A clinical trial to evaluate the worth of preoperative multimodality therapy in patients with operable carcinoma of the rectum: a progress report of National Surgical Breast and Bowel Project Protocol R-03. Dis Colon Rectum 1997:40:131-9.

8. Wasmuth HH, Rekstad LC, Trano G. The outcome and the frequency of pathological complete response after neoadjuvant radiotherapy in curative resections for advanced rectal cancer: a population-based study. Colorectal Dis 2016;18:67-72.

9. Yarnold J. Molecular aspects of cellular responses to radiotherapy. Radiother Oncol 1997;44:1-7.

10. Haboubi NY, El-Zammar O, O'Dwyer ST, James RJ. Radiation bowel disease: pathogenesis and management. Colorectal Dis 2000; 2:322-9.

11. Metheetrairut C, Slack FJ. MicroRNAs in the ionizing radiation response and in radiotherapy. Curr Opin Genet Dev 2013;23:12-9.

12. Gay HA, Barthold HJ, O'Meara E, et al. Pelvic normal tissue contouring guidelines for radiation therapy: a Radiation Therapy On- 
cology Group consensus panel atlas. Int J Radiat Oncol Biol Phys 2012;83:e353-62.

13. Verma $C$, Eremin JM, Robins $A$, et al. Abnormal T regulatory cells (Tregs: FOXP3+, CTLA-4+), myeloid-derived suppressor cells (MDSCs: monocytic, granulocytic) and polarised T helper cell profiles (Th1, Th2, Th17) in women with large and locally advanced breast cancers undergoing neoadjuvant chemotherapy (NAC) and surgery: failure of abolition of abnormal treg profile with treatment and correlation of treg levels with pathological response to NAC. J Transl Med 2013;11:16.

14. Rödel C, Graeven U, Fietkau R, et al. Oxaliplatin added to fluorouracil-based preoperative chemoradiotherapy and postoperative chemotherapy of locally advanced rectal cancer (the German CAO/ARO/AIO-04 study): final results of the multicentre, open-label, randomised, phase 3 trial. Lancet Oncol 2015; 16:979-89.

15. Habr-Gama A, Perez RO, Nadalin W, et al. Operative versus nonoperative treatment for stage 0 distal rectal cancer following chemoradiation therapy: long-term results. Ann Surg 2004; 240:711-8.

16. Glynne-Jones R, Wallace M, Livingstone Jl, Meyrick-Thomas J. Complete clinical response after preoperative chemoradiation in rectal cancer: is a "wait and see" policy justified? Dis Colon Rectum 2008;51:10-20.

17. Maas M, Beets-Tan RG, Lambregts DM, et al. Wait-and-see policy for clinical complete responders after chemoradiation for rectal cancer. J Clin Oncol 2011;29:4633-40.

18. Maas M, Lambregts DM, Nelemans PJ, et al. Assessment of clinical complete response after chemoradiation for rectal cancer with digital rectal examination, endoscopy, and MRI: selection for organ-saving treatment. Ann Surg Oncol 2015;22:3873.

19. Xiao $L, Y u X_{1}$ Deng $W$, et al. Pathological assessment of rectal cancer after neoadjuvant chemoradiotherapy: distribution of residual cancer cells and accuracy of biopsy. Sci Rep 2016;6:34923.

20. Perez RO, Habr-Gama A, Gama-Rodrigues J, et al. Accuracy of positron emission tomography/computed tomography and clinical assessment in the detection of complete rectal tumor regression after neoadjuvant chemoradiation: long-term results of a prospective trial (National Clinical Trial 00254683). Cancer 2012;118:3501-11.

21. Jones DJ, Zaloudik J, James RD, Haboubi N, Moore M, Schofield PF. Predicting local recurrence of carcinoma of the rectum after preoperative radiotherapy and surgery. Br J Surg 1989;76:11725.

22. Harnden P, Shelley MD, Naylor B, Coles B, Mason MD. Does the extent of carcinoma in prostatic biopsies predict prostate-specific antigen recurrence? A systematic review. Eur Urol 2008;54:728-
39.

23. Mandard AM, Dalibard F, Mandard JC, et al. Pathologic assessment of tumor regression after preoperative chemoradiotherapy of esophageal carcinoma. Clinicopathologic correlations. Cancer 1994;73:2680-6.

24. Dworak O, Keilholz L, Hoffmann A. Pathological features of rectal cancer after preoperative radiochemotherapy. Int J Colorectal Dis 1997;12:19-23.

25. Wheeler JM, Warren BF, Mortensen NJ, et al. Quantification of histologic regression of rectal cancer after irradiation: a proposal for a modified staging system. Dis Colon Rectum 2002;45:10516.

26. Bateman $A C$, Jaynes $E_{1}$ Bateman AR. Rectal cancer staging post neoadjuvant therapy: how should the changes be assessed? Histopathology 2009;54:713-21.

27. Ryan R, Gibbons D, Hyland JM, et al. Pathological response following long-course neoadjuvant chemoradiotherapy for locally advanced rectal cancer. Histopathology 2005;47:141-6.

28. Amin MB, Greene FL, Edge SB, et al. The eighth edition AJCC Cancer Staging Manual: continuing to build a bridge from a population-based to a more "personalized" approach to cancer staging. CA Cancer J Clin 2017;67:93-9.

29. Kim SH, Chang HJ, Kim DY, et al. What is the ideal tumor regression grading system in rectal cancer patients after preoperative chemoradiotherapy? Cancer Res Treat 2016;48:998-1009.

30. Fokas E, Fietkau R, Hartmann A, et al. Neoadjuvant rectal score as individual-level surrogate for disease-free survival in rectal cancer in the CAO/ARO/AIO-04 randomized phase III trial. Ann Oncol 2018;29:1521-7.

31. National Cancer Comprehensive Network. NCCN Guidelines for Patients [Internet]. Plymouth Meeting, PA: National Cancer Comprehensive Network; 2019 [cited 2020 May 1]. Available from: https://www.nccn.org/patients/.

32. Bujko K, Nowacki MP, Nasierowska-Guttmejer A, et al. Sphincter preservation following preoperative radiotherapy for rectal cancer: report of a randomised trial comparing short-term radiotherapy vs. conventionally fractionated radiochemotherapy. Radiother Oncol 2004;72:15-24.

33. Bujko K, Nowacki MP, Nasierowska-Guttmejer A, Michalski W, Bebenek M, Kryj M. Long-term results of a randomized trial comparing preoperative short-course radiotherapy with preoperative conventionally fractionated chemoradiation for rectal cancer. Br J Surg 2006;93:1215-23.

34. Simpson GS, Eardley N, McNicol F, Healey P, Hughes M, Rooney PS. Circumferential resection margin (CRM) positivity after MRI assessment and adjuvant treatment in 189 patients undergoing rectal cancer resection. Int J Colorectal Dis 2014;29:585-90. 
35. Pawlik TM, Keyomarsi K. Role of cell cycle in mediating sensitivity to radiotherapy. Int J Radiat Oncol Biol Phys 2004;59:928-42.

36. Sauer R, Becker $H$, Hohenberger W, et al. Preoperative versus postoperative chemoradiotherapy for rectal cancer. N Engl J Med 2004;351:1731-40.

37. Colorectal Cancer Collaborative Group. Adjuvant radiotherapy for rectal cancer: a systematic overview of 8,507 patients from 22 randomised trials. Lancet 2001;358:1291-304.

38. Gollins S, West N, Sebag-Montefiore D, et al. Preoperative chemoradiation with capecitabine, irinotecan and cetuximab in rectal cancer: significance of pre-treatment and post-resection RAS mutations. Br J Cancer 2017;117:1286-94.

39. Salmo E, El-Dhuwaib Y, Haboubi NY. Histological grading of tumour regression and radiation colitis in locally advanced rectal cancer following neoadjuvant therapy: a critical appraisal. Colorectal Dis 2011;13:1100-6.

40. Nagtegaal ID, Marijnen CA, Kranenbarg EK, et al. Short-term preoperative radiotherapy interferes with the determination of pathological parameters in rectal cancer. J Pathol 2002;197:207.

41. Maas M, Nelemans PJ, Valentini V, et al. Long-term outcome in patients with a pathological complete response after chemoradiation for rectal cancer: a pooled analysis of individual patient data. Lancet Oncol 2010;11:835-44.

42. Kong JC, Guerra GR, Warrier SK, et al. Prognostic value of tumour regression grade in locally advanced rectal cancer: a systematic review and meta-analysis. Colorectal Dis 2018;20:574-85.

43. Battersby NJ, Dattani M, Rao S, et al. A rectal cancer feasibility study with an embedded phase III trial design assessing magnetic resonance tumour regression grade ( $\mathrm{mrTRG}$ ) as a novel bio- marker to stratify management by good and poor response to chemoradiotherapy (TRIGGER): study protocol for a randomised controlled trial. Trials 2017;18:394.

44. Eriksen AH, Sorensen FB, Andersen RF, Jakobsen A, Hansen TF. Association between the expression of microRNAs and the response of patients with locally advanced rectal cancer to preoperative chemoradiotherapy. Oncol Lett 2017;14:201-9.

45. D'Angelo E, Zanon C, Sensi F, et al. miR-194 as predictive biomarker of responsiveness to neoadjuvant chemoradiotherapy in patients with locally advanced rectal adenocarcinoma. J Clin Pathol 2018;71:344-50.

46. Luo J, Liu L, Zhou N, et al. miR-519b-3p promotes responsiveness to preoperative chemoradiotherapy in rectal cancer patients by targeting ARID4B. Gene 2018;655:84-90.

47. Yu J, Li N, Wang $X$, et al. Circulating serum microRNA-345 correlates with unfavorable pathological response to preoperative chemoradiotherapy in locally advanced rectal cancer. Oncotarget 2016;7:64233-43.

48. Martinez-Useros J, Moreno I, Fernandez-Acenero MJ, et al. The potential predictive value of DEK expression for neoadjuvant chemoradiotherapy response in locally advanced rectal cancer. BMC Cancer 2018;18:144.

49. Peng H, You K, Zhang R, et al. Predictive value of APAF-1 and COX-2 expression in pathologic complete response to neoadjuvant chemoradiotherapy for patients with locally advanced rectal adenocarcinoma. Oncotarget 2016;7:35233-40.

50. Repetto O, De Re V, De Paoli A, et al. Identification of protein clusters predictive of tumor response in rectal cancer patients receiving neoadjuvant chemo-radiotherapy. Oncotarget 2017; 8:28328-41. 